Bull. Austral. Math. Soc.

$05 \mathrm{C} 80,68 \mathrm{Q} 35$

VOL. 39 (1988) [389-396]

\title{
THE BISECTION WIDTH OF CUBIC GRAPHS
}

\author{
L.H. Clark and R.C. Entringer
}

\begin{abstract}
For a graph $G$, define the bisection width $b w(G)$ of $G$ as $\min \left\{e_{G}(A, B):\{A, B\}\right.$ partitions $V(G)$ with $\|A|-| B\| \leqslant 1\}$ where $e_{G}(A, B)$ denotes the number of edges in $G$ with one end in $A$ and one end in $B$. We show almost every cubic graph $G$ of order $n$ has $b w(G) \geqslant n / 11$ while every such graph has $b w(G) \leqslant(n+138) / 3$. We also show that almost every $r$-regular graph $G$ of order $n$ has $b w(G) \geqslant c_{r} n$ where $c_{r} \rightarrow r / 4$ as $r \rightarrow \infty$. Our last result is asym totically correct.
\end{abstract}

\section{INTRODUCTION}

For a graph $G$, define the bisection width $b w(G)$ of $G$ by

$$
b w(G)=\min \left\{e_{G}(A, B):\{A, B\} \text { partitions } V(G) \text { with }|| A|-| B|| \leqslant 1\right\}
$$

where $e_{G}(A, B)$ denotes the number of edges in $G$ with one end in $A$ and one end in $B$.

The problem of finding the bisection width of a graph is of fundamental importance in many divide-and-conquer stratagems and, as such, is the subject of an extensive literature. (See $[4,9,10,13,15,18]$ for general results and $[6,11]$ for results regarding VLSI design.)

Unfortunately, the bisection problem for graphs, in general, is NP-complete [12] and remains so for $r$-regular graphs [0]. Polynomial-time algorithms which give exact solutions are known only for trees and bounded-width planar graphs [9] while polynomial-time algorithms which give approximate solutions may give solutions which are far from exact [18]. Consequently, heuristic algorithms which hopefully give nearly exact solutions most of the time have been developed in $[9,13,14,16,18]$.

In [9] a method was given for transforming a regular graph $G$ of order $n$ into a cubic graph $G^{*}$ of order $\Theta\left(n^{6}\right)$ so that any minimum bisection of $G^{*}$ uses only edges of $G$. As a result, we content ourselves mainly with an examination of cubic graphs. As usual, we say that almost every graph has a property $Q$ provided the probability that a graph of order $n$ has property $Q$ tends to 1 as $n \rightarrow \infty$.

We show that almost every cubic graph $G$ of order $n$ has $b w(G) \geqslant n / 11$ while every such graph has $b w(G) \leqslant(n+138) / 3$. We also show that almost every $r$-regular

Received 22 July, 1988

Copyright Clearance Centre, Inc. Serial-fee code: 0004-9729/88 \$A2.00+0.00. 
graph $G$ of order $n$ has $b w(G) \geqslant c_{r} n$ where $c_{r} \rightarrow r / 4$ as $r \rightarrow \infty$. (Note that absolute lower bounds for the bisection width of a graph are not particularly informative, since they must be nearly zero.)

Our notation and terminology follows Bollobás [7].

\section{An Upper Bound for the Bisection Width of a Cubic Graph}

We give now an upper bound for the bisection width of a cubic graph.

TheOREM 1. Every cubic graph $G$ of order $n$ has $b w(G) \leqslant(n+138) / 3$.

Proof: Let $\{A, B\}$ be an equisized partition of $V(G)$ with $b w(G)=e_{G}(A, B)$. Set $A_{i}=\left\{v \in A: e_{G}(v, B)=i\right\}$ for $0 \leqslant i \leqslant 3$ and $A_{1 i}=\left\{v \in A_{1}: e_{G}\left(v, A-A_{1}\right)=i\right\}$ for $0 \leqslant i \leqslant 2$. (Define $B_{i}$ and $B_{1 i}$ similarly.)

Suppose $x \in A_{3}$ and $y \in B_{1} \cup B_{2} \cup B_{3}$ with $x y \notin E(G)$; exchanging $x$ with $y$ shows $\{A, B\}$ is not an optimal partition, which is a contradiction. Consequently, $\left|B_{1} \cup B_{2} \cup B_{3}\right| \leqslant 3$ and $b w(G) \leqslant 9 \leqslant(n+138) / 3$. We assume $\left|A_{3}\right|=\left|B_{3}\right|=0$.

Suppose $\left|B_{2}\right| \geqslant 4$. When $\left|A_{2}\right| \neq 0$, there exists $x \in A_{2}$ and $y \in B_{2}$ with $x y \notin$ $E(G)$; exchanging $x$ with $y$ shows $\{A, B\}$ is not an optimal partition. Consequently, $\left|A_{2}\right|=0$. When $G\left[A_{10} \cup A_{11}\right]$ is empty, we have $\left|A_{10} \cup A_{11}\right| \leqslant 1$. Then

$$
3\left|A_{0}\right| \geqslant \epsilon_{G}\left(A_{0}, A_{1}\right) \geqslant 2\left|A_{1}\right|-2
$$

so that

$$
n / 2=\left|A_{0}\right|+\left|A_{1}\right| \geqslant\left(5\left|A_{1}\right|-2\right) / 3
$$

and

$$
b w(G) \leqslant(3 n+4) / 10 \leqslant(n+138) / 3 .
$$

When $G\left[A_{10} \cup A_{11}\right]$ is nonempty, there exist an edge $x_{1} x_{2}$ in $G\left[A_{10} \cup A_{11}\right]$ and $y_{1}, y_{2} \in$ $B_{2}$ with $e_{G}\left(\left\{x_{1}, x_{2}\right\},\left\{y_{1}, y_{2}\right\}\right)=0$; exchanging $\left\{x_{1}, x_{2}\right\}$ with $\left\{y_{1}, y_{2}\right\}$ shows $\{A, B\}$ is not an optimal partition. We assume $\left|A_{2}\right|,\left|B_{2}\right| \leqslant 3$.

Denote a path (cycle) of order $n$ by $P_{n}\left(C_{n}\right)$. Let

$$
\begin{aligned}
& a=\max \left|\left\{E_{1}, \ldots, E_{t}\right\}\right| \text { where }\left\{E_{1}, \ldots, E_{t}\right\} \text { is a set of } \\
& \text { vertex-disjoint subgraphs of } G[A] \text { and each }
\end{aligned}
$$$$
E_{i} \cong P_{3} \subseteq G\left[A_{10} \cup A_{11}\right] \text { or }
$$

$\cong C_{3} \subseteq G\left[A_{0} \cup A_{11}\right]$ with precisely one vertex in $A_{0}$ or

$\cong C_{4} \subseteq G\left[A_{0} \cup A_{10} \cup A_{11}\right]$ with precisely one vertex in

$A_{0}$ and precisely one vertex in $A_{10}$ or

$\cong P_{5} \subseteq G\left[A_{0} \cup A_{10} \cup A_{11}\right]$ with only the centre vertex

in $A_{0}$ and 
let $A_{j}^{*}=\bigcup\left\{V\left(E_{i}\right) \cap A_{j}: 1 \leqslant i \leqslant a\right\}$ for $0 \leqslant j \leqslant 1$. (Define $b,\left\{F_{1}, \ldots, F_{t}\right\}, B_{j}^{*}$ for $0 \leqslant j \leqslant 1$ similarly.)

Claim. $\min \{a, b\} \leqslant 5$.

Suppose $a, b \geqslant 6$. Choose $e_{G}\left(E_{i}, F_{j}\right)=0$ with ||$E_{i}|-| F_{j}||$ as large as possible, say $\left|E_{i}\right| \geqslant\left|F_{j}\right|$. When $\left|E_{i}\right|=\left|F_{j}\right|$; exchanging $E_{i}$ with $F_{j}$ shows $\{A, B\}$ is not an optimal partition. When $\left|E_{i}\right|=\left|F_{j}\right|+1$; exchanging $E_{i}^{\prime}$ with $F_{j}$, where $E_{i}^{\prime}$ is the subgraph of $E_{i}$ contained in $G\left[A_{1}\right]$, shows $\{A, B\}$ is not an optimal partition. When $\left|E_{i}\right|=\left|F_{j}\right|+2$ then $\left|E_{i}\right|=5$ and $\left|F_{j}\right|=3$. Since $b \geqslant 6$, there exist $F_{k} \neq F_{j}$ with $e_{G}\left(E_{i}, F_{k}\right)=0$. By the above, $\left|F_{k}\right|=3$; exchanging $E_{i}$ with $F_{j} \cup F_{k}^{\prime}$, where $F_{k}^{\prime}$ is a subpath of order 2 contained in $G\left[B_{1}\right]$, shows $\{A, B\}$ is not an optimal partition.

We assume $a \leqslant 5$ so that $\left|A_{0}^{*}\right| \leqslant 5$ and $\left|A_{1}^{*}\right| \leqslant 20$.

Claim. $\left|A_{10}\right| \leqslant 25$.

Note that $G\left[A_{10} \cup A_{11}\right]$ is a vertex-disjoint set of paths and cycles when $\mid A_{10} \cup$ $A_{11} \mid \neq 0$, since $\delta\left(G\left[A_{10} \cup A_{11}\right]\right)=1$ and $\Delta\left(G\left[A_{10} \cup A_{11}\right]\right)=2$. Consequently, $\left|A_{10}\right| \leqslant$ 25 since $a \leqslant 5$ (after breaking paths and cycles apart if necessary).

Let $A_{1}^{\prime}=\left\{w \in A_{1}-A_{1}^{*}: v w \in E(G)\right.$ for some $\left.v \in A_{1}^{*}\right\}$. Clearly, $\left|A_{1}^{\prime}\right| \leqslant 2 \cdot 5=10$. Set $\left|A_{12}\right|=c\left|A_{1}\right|$ where $c \in[0,1]$.

Then

$$
\left|A_{11}\right|+\left|A_{12}\right| \geqslant\left|A_{1}\right|-25
$$

so that

$$
\left|A_{11}\right| \geqslant(1-c)\left|A_{1}\right|-25
$$

Now

$$
3\left|A_{0}\right| \geqslant e_{G}\left(A_{0}, A_{1}\right) \geqslant\left|A_{11}\right|+2\left|A_{12}\right|-3
$$

so that

$$
\left|A_{0}\right| \geqslant\left[(1+c)\left|A_{1}\right|-28\right] / 3
$$

Then

$$
n / 2 \geqslant\left|A_{0}\right|+\left|A_{1}\right| \geqslant\left[(4+c)\left|A_{1}\right|-28\right] / 3
$$

so that

$$
\left|A_{1}\right| \leqslant(3 n+56) / 2(4+c)
$$

and

$$
b w(G) \leqslant 6+\left|A_{1}\right| \leqslant 6+(3 n+56) / 2(4+c) .
$$

Also

$$
\begin{aligned}
\left|A_{11}\right|-\left|A_{1}^{*}\right|-\left|A_{1}^{\prime}\right|-5 & \leqslant\left|A_{11}-\left(A_{1}^{*} \cup A_{1}^{\prime}\right)\right|-5 \\
& \leqslant\left|A_{0}-A_{0}^{*}\right|=\left|A_{0}\right|-\left|A_{0}^{*}\right|,
\end{aligned}
$$


by the maximality of $a$, so that

$$
\left|A_{0}\right| \geqslant\left|A_{11}\right|-35 \geqslant(1-c)\left|A_{1}\right|-60 .
$$

Then

$$
n / 2 \geqslant\left|A_{0}\right|+\left|A_{1}\right| \geqslant(2-c)\left|A_{1}\right|-60
$$

so that

$$
\left|A_{1}\right| \leqslant(n+120) / 2(2-c)
$$

and

$$
b w(G) \leqslant 6+\left|A_{1}\right| \leqslant 6+(n+120) / 2(2-c) .
$$

Consequently,

$$
\begin{aligned}
b w(G) & \leqslant \min \{6+(3 n+56) / 2(4+c), 6+(n+120) / 2(2-c)\} \\
& \leqslant(n+138) / 3
\end{aligned}
$$

since the above minimum is at most $(n+138) / 3$ for $n \geqslant 184$ and at most $6+$ $(3 n+56) / 8 \leqslant(n+138) / 3$ for $n \leqslant 182$.

Remark. In general, if $\{A, B\}$ is a partition of the vertices of an $r$-regular graph $G$ of order $n$ with $b w(G)=e_{G}(A, B)$, one would hope that either $G[A]$ or $G[B]$ contains a small number of forbidden subgraphs (see definition of $a, b$ in Theorem 1 ) which, in turn, impose structure on $G[A]$ or $G[B]$ and give $b w(G) \leqslant c_{r} n+O(1)$ for some $c_{r}<r / 4$. At present we have only the result of Goldberg and Gardner [13] that, for any such graph $G, b w(G) \leqslant r\left(n+\varepsilon_{n}\right) / 4$ where $\varepsilon_{n}=1$ for odd $n$ and $\varepsilon_{n}=n /(n-1)$ for even $n$. There are, however, limitations on how small the ratio $b w(G) / n$ can be made for $r$-regular graphs $G$ of order $n$.

An $r$-regular graph $G$ of order $n$ is an $(n, r, c)$-expander if $|N(X)-X| \geqslant c|X|$ for all $X \subseteq V(G)$ with $|X| \leqslant n / 2$. (These and similar graphs have an extensive literature; see the references in [1].) Clearly, any $(n, r, c)$-expander $G$ has $b w(G) \geqslant c[n / 2]$.

Let $\lambda_{1}(G)$ denote the second largest eigenvalue of the adjacency matrix of $G$ in absolute value. Note that $0<\lambda_{1}(G)<r$ when $G$ is connected. Alon and Milman [3] have shown that any $r$-regular graph $G$ of order $n$ is an $\left(n, r,\left(r-\lambda_{1}(G)\right) / 2 r\right)$ expander while Alon and Boppana [2] (see also [17]) have shown that $\underset{n \rightarrow \infty}{\lim } \lambda_{1}\left(G_{n}\right) \geqslant$ $2 \sqrt{r-1}$ for any sequence $\left\{G_{n}\right\}$ of such graphs. Lubotzky, Phillips and Sarnak [17] have shown this last result asymptotically correct by constructing infinite families of $r$-regular graphs $G$ with $\lambda_{1}(G) \leqslant 2 \sqrt{r-1}$ for all primes $r-1 \equiv 1(\bmod 4)$.

The above results imply that any $r$-regular graph $G$ of large order $n$ has $b w(G) \geqslant$ $c n$ where $c$, unfortunately, is rather small. We improve this by showing that almost every $r$-regular graph $G$ of order $n$ has $b w(G) \geqslant c_{r} n$ where $c_{r} \rightarrow r / 4$ as $r \rightarrow \infty$. 


\section{A LOWER BOUND FOR THE BISECTION WIDTH OF Almost Every Cubic Graph}

Bender and Canfield [5] gave the first formula for the asymptotic number of labelled $r$-regular graphs of order $n$. Bollobás [8] gave a simpler proof of the same formula that, more importantly, contained a model for the set of regular graphs which can be used to study labelled random regular graphs. We describe now this model.

Let $r n$ be even and $q=r n / 2$. Let $V=V_{1} \cup \cdots \cup V_{n}$ be a disjoint union of $r n$ labelled vertices where $\left|V_{i}\right|=r$ for $1 \leqslant i \leqslant n$. A configuration is a 1-regular graph with vertex set $V$. Denote the set of configurations by $\Phi=\Phi(n, r)$. Clearly,

$$
|\Phi|=(r n) ! / 2^{q} q ! \text {. }
$$

A configuration is good if when we shrink each set $V_{i}$ to a vertex $v_{i}$ we obtain a simple graph. Denote the set of good configurations by $\Omega=\Omega(n, r)$ and the set of simple $r$-regular graphs with vertex set $\left\{v_{1}, \ldots, v_{n}\right\}$ by $\mathcal{G}_{n}^{(r)}$. Clearly,

$$
|\Omega|=(r !)^{n}\left|\mathcal{G}_{n}^{(r)}\right|
$$

Now regard $\Phi$ as a probability space where $P(F)=|\Phi|^{-1}$ for any configuration $F$. Bollobás [8] showed that

$$
P(\text { configuration } F \text { is good }) \rightarrow e^{\left(1-r^{2}\right) / 4} \quad(n \rightarrow \infty)
$$

and, hence,

$$
\left|\mathcal{G}_{n}^{(r)}\right| \sim e^{\left(1-r^{2}\right) / 4}|\Phi| /(r !)^{n} \quad(n \rightarrow \infty)
$$

Finally regard $\mathcal{G}_{n}^{(r)}$ as a probability space where $P(G)=\left|\mathcal{G}_{n}^{(r)}\right|^{-1}$ for any $r$-regular graph $G$ with vertex set $\left\{v_{1}, \ldots, v_{n}\right\}$. An immediate consequence of the preceding is that if the probability that a configuration has a certain property tends to 1 as $n \rightarrow \infty$ then the probability that an $r$-regular graph has the corresponding property also tends to 1 as $n \rightarrow \infty$.

For $r \geqslant 3$, let $c=c_{r}$ be the unique real number in $(0, r / 4)$ with $2^{(2-r)} r^{r}=$ $(2 c)^{2 c}(r-2 c)^{(r-2 c)}$. (The constant exists since $x^{x}(r-x)^{r-x}$ monotonically decreases on $[0, r / 2]$.$) Note that c_{3}=.0922357 \ldots \in(1 / 11,1 / 10)$. We denote $t(t-1) \cdots$ $(t-k+1)$ by $(t)_{k}$.

We give now

Theorem 2. Almost every cubic graph $G$ of order $n$ has $b w(G) \geqslant n / 11$.

Proof: Let $n=2 m$. Fix a partition $\{A, B\}$ of $\{1, \ldots, n\}$ with $|A|=|B|=m$. Let $V_{A}=\bigcup\left\{V_{i}: i \in A\right\}$ (Define $V_{B}$ similarly). Note that the event $e_{F}\left(V_{A}, V_{B}\right)=j$ 
is a nonempty subset of $\Phi$ if and only if $3 \mathrm{~m}$ and $j$ have the same parity. Put $p_{j}=$ $(3 m-j) / 2$. Then

$$
\begin{aligned}
P\left(e_{F}\left(V_{A}, V_{B}\right)=j\right) & =\frac{(3 m)_{j}^{2}}{j !}\left[\frac{(3 m-j) !}{2^{p_{j}} p_{j} !}\right]^{2}|\Phi|^{-1} \\
& =\frac{[(3 m) !]^{3} 2^{j}}{j !\left[p_{j} !\right]^{2}(r n) !}
\end{aligned}
$$

where the left factor of (1) is the number of ways of labelling the ends of the $j$ edges between $V_{A}$ and $V_{B}$ and the middle factor of (1) is the number of ways of completing the 1-factor in both $V_{A}$ and $V_{B}$.

For $j \geqslant 2$, we have

$$
P\left(e_{F}\left(V_{A}, V_{B}\right)=j-2\right)=\frac{j(j-1)}{(3 m-j+2)^{2}} P\left(e_{F}\left(V_{A}, V_{B}\right)=j\right)
$$

where $j(j-1) /(3 m-j+2)^{2}$ increases with $j$. For even $3 m$ and $2 k \leqslant\left\lfloor c_{3} n\right\rfloor$, we have

$$
\begin{aligned}
P\left(e_{F}\left(V_{A}, V_{B}\right) \leqslant 2 k\right) & =\sum_{\text {even } j \leqslant 2 k} P\left(e_{F}\left(V_{A}, V_{B}\right)=j\right) \\
& \leqslant P\left(e_{F}\left(V_{A}, V_{B}\right)=2 k\right)\left(1+\alpha+\cdots+\alpha^{k}\right),
\end{aligned}
$$

where $\alpha=2 k(2 k-1) /(3 m-2 k+2)^{2}$. Since $\alpha \leqslant\left(2 c_{3} / 3-2 c_{3}\right)^{2} \leqslant 1 / 2$, we have

$$
P\left(e_{F}\left(V_{A}, V_{B}\right) \leqslant 2 k\right) \leqslant 2 P\left(e_{F}\left(V_{A}, V_{B}\right)=2 k\right)
$$

Then

$$
\begin{aligned}
P(b w(F) \leqslant 2 k) & =P\left(e_{F}\left(V_{A}, V_{B}\right) \leqslant 2 k \text { for some }\{A, B\}\right) \\
& \leqslant \sum_{\{A, B\}} P\left(e_{F}\left(V_{A}, V_{B}\right) \leqslant 2 k\right) \\
& \leqslant\left(\begin{array}{c}
n \\
m
\end{array}\right) \frac{[(3 m) !]^{3} 2^{2 k}}{(2 k) !\left[p_{2 k} !\right]^{2}(3 n) !}
\end{aligned}
$$

From $\left(\begin{array}{l}n \\ m\end{array}\right)=O\left(2^{n} m^{-1 / 2}\right)$ and Stirling's Formula, we obtain

$$
P(b w(F) \leqslant 2 k)=O\left(\frac{2^{-m} 3^{3 m} m^{3 m+1 / 2}}{(2 k)^{2 k+1 / 2}(3 m-2 k)^{3 m-2 k+1}}\right) .
$$

Now write $2 k=2 c m \leqslant\left\lfloor c_{3} n\right\rfloor$ and we have

$$
P(b w(F) \leqslant c n)=O\left(n^{-1}\right) .
$$


For odd $3 m$, a similar calculation with $2 k$ replaced by $2 k+1$ gives the same result. Then

$$
P\left(b w(F) \leqslant\left\lfloor c_{3} n\right\rfloor\right) \rightarrow 0 \quad(n \rightarrow \infty)
$$

and, consequently,

$$
P\left(b w\left(G \in \mathcal{G}_{n}^{(3)}\right) \geqslant c_{3} n\right) \rightarrow 1 \quad(n \rightarrow \infty) .
$$

Remark. In general, a similar calculation shows that

$$
P\left(b w\left(G \in \mathcal{G}_{n}^{(r)}\right) \geqslant c_{r} n\right) \rightarrow 1 \quad(n \rightarrow \infty)
$$

Since $(2 d)^{2 d}(1-2 d)^{(1-2 d)}$ monotonoically decreases to $1 / 2$ on $[0,1 / 4]$, we have $(2 d)^{2 d}(1-2 d)^{(1-2 d)} \geqslant 2^{2 / r} / 2$ for fixed $d \in(0,1 / 4)$ and all sufficiently large $r$. Consequently, $c_{r} \geqslant r d$ so that $c_{r} \rightarrow r / 4$ as $r \rightarrow \infty$. We summarize this now.

Theorem 3. Almost every $r$-regular graph $G$ of order $n$ has $b w(G) \geqslant c_{r} n$. Moreover, $c_{r} \rightarrow r / 4$ as $r \rightarrow \infty$.

In view of the upper bound for the bisection width given by Goldberg and Gardner [13], this last result is asymptotically correct.

\section{REFERENCES}

[1] N. Alon, 'Eigenvalues and expanders', Combinatorica 6 (1986), 83-96.

[2] N. Alon and R. Boppana (preprint).

[3] N. Alon and V.D. Milman, ' $\lambda_{1}$, Isoperimetric inequalities for graphs, and superconcentrators', $J$. Combin. Theory Ser. B 38 (1985), 73-88.

[4] E.R. Barnes, 'Partitioning the nodes of a graph', in Graph Theory with Applications to Algorithms and Computer Science, ed. Y. Alavi, et al, pp. 57-72 (Wiley Interscience, New York, 1985).

[5] E.A. Bender and E.R. Canfield, 'The asymptotic number of labelled graphs with given degree sequence', J. Combin. Theory Ser. A 24 (1978), 296-307.

[6] S.N. Bhatt and F.T. Leighton, 'A framework for solving VLSI graph layout problems', J. Comput. System Sci. 28 (1984), 300-343.

[7] B. Bollobás, Graph Theory, An Introductory Course (Springer-Verlag, Berlin, Heidelberg, New York, 1979).

[8] B. Bollobás, 'A probabilistic proof of an asymptotic formula for the number of labelled regular graphs', European J. Combin. 1 (1980), 311-316.

[ө] T.N. Bui, S. Chaudhuri, F.T. Leighton and M. Sipser, 'Graph bisection algorithms with good average case behaviour', Combinatorica 7 (1987), 171-191.

[10] W.E. Donath and A.J. Hoffman, 'Lower bounds for the partitioning of graphs', $I B M J$. Res. Develop. 17 (1973), 420-425.

[11] A.E. Dunlop and B.W. Kernighan, 'A procedure for placement of standard-cell VLSI circuits', IEEE Transactions on Computer-Aided Design CAD-4 (1985), 92-98. 
[12] M.R. Garey and D.S. Johnson, Computers and Intractability: A guide to the thoery of $N P$-completeness (W.M. Freeman, New York, 1979).

[13] M.K. Goldberg and R. Gardner, 'On the minimal cut problem', in Progress in Graph Theory, ed. J.A. Bondy et al, pp. 295-305 (Academic Press, New York, 1984).

[14] M.K. Goldberg, S. Lath and J.W. Roberts, 'Heuristics for the graph bisection problem', (preprint).

[15] M.K. Goldberg and J.F. Lynch, 'Lower and upper bounds for the bisection width of a random graph', Congress. Numer. 49 (1985), 19-25.

[16] B.W. Kernighan and S. Lin, 'An efficient heuristic procedure for partitioning graphs', Bell System Technical J. 49 (1970), 291-307.

[17] A. Lubotzky, R. Phillips and P. Sarnak, 'Ramanujan graphs', Combinatorica (to appear).

[18] R.M. MacGregor, On partitioning a graph: a theoretical and empirical study. (Ph.D thesis, University of California, Berkeley, 1978).

\section{L.H. Clark,}

Department of Mathematics,

The University of New Mexico,

Albuquerque, NM. 87131

United States of America.
R. Entringer,

Department of Mathematics,

The University of New Mexico,

Albuquerque, NM. 87131

United States of America. 